\title{
Assessment of Genetic Variability, Heritability and Genetic Advance for Yield in Advanced Breeding Line (Oryza sativa L.) of Low Land Rice in Meghalaya
}

\author{
Ashish Rai ${ }^{1}$, Mayank Rai ${ }^{2}$, Bidisha Borpatragohain ${ }^{3}$ and Shivendra kumar ${ }^{4}$ \\ ${ }^{1}$ Department of Plant Breeding and Genetics, ${ }^{3}$ Department of Soil Science, ${ }^{4}$ Department of \\ Biotechnology, Dr. Rajendra Prasad Central Agricultural University, Pusa, Bihar \\ ${ }^{2}$ Department of Genetics and Plant Breeding, \\ Central Agricultural University, Imphal, Manipur \\ *Corresponding author
}

A B S T R A C T

\begin{tabular}{l} 
Ke y w o r d s \\
Genotypic \\
coefficient of \\
variation, Genetic \\
improvement, \\
Genetic variability, \\
Heritability and \\
phenotypic \\
coefficient of \\
variation \\
\hline Article Info \\
\hline $\begin{array}{l}\text { Accepted: } \\
\text { 05 February } 2020 \\
\text { Available Online: } \\
\text { 10 March } 2020\end{array}$ \\
\hline
\end{tabular}

\begin{abstract}
The genetic parameters were studied to generate information on genetic variability, heritability and genetic advance among 22 advanced breeding lines including two checks at the experimental Farm of College of post graduate studies, CAU (Imphal), Umiam, Meghalaya during Kharif 2017. Analysis of variance indicated the existence of significant differences among the genotypes for most of the characters. High Phenotypic Coefficient of variation $(\mathrm{PCV})$ and Genotypic Coefficient of Variation $(\mathrm{GCV})$ values were recorded for number of grain per panicle and spikelet per plant which suggests the possibility of improving this trait through selection. The low magnitude of difference between phenotypic and genotypic coefficients of variations were recorded for characters such as days to $50 \%$ flowering, leaf length and leaf width indicating limited influence of environment in the expression of this trait. Thus, selection based on phenotypic expression of the trait would be effective for genetic improvement. High heritability in broad sense values indicate that the traits under study are less influenced by environment in their expression. Therefore, the quantitative traits are highly heritable. However, highest heritability was recorded for leaf length and leaf width. Moderate heritability estimates were observed for number of panicles per plant, spikelets per panicle, grains per panicle, and spikelet fertility.
\end{abstract}

\section{Introduction}

The genus Oryza consists of two cultivated species Oryza sativa (Asian species) and Oryza glaberrima (African species). Rice (Oryza sativa) is the primary food source for more than a third of the world's population.
South Asia is considered to be one of the major centres for rice domestication and is also known as the food bowl of Asia. Asia accounts for over $90 \%$ of the world's production of rice, which is mainly contributed by China, India and Indonesia. Among all the Asian countries, India is the 
prominent rice growing country accounting for about $20 \%$ of all world rice production. India is home to wide varieties of rice cultivars, landraces and many lesser known varieties that have been under cultivation since ages by farmers. In India rice is the major crop grown at $43.57 \mathrm{M}$ ha with an average production of 104.32 million tonnes and productivity of $2.39 \mathrm{t} / \mathrm{ha}$. In Meghalaya rice is grown in more than $42 \%$ of total arable area, but is having average production of 2.32 lakh tonnes and productivity of $2.12 \mathrm{t} / \mathrm{ha}$. During the post green revolution period due to introduction of improved varieties, rice yield in North Eastern hill region has been enhanced by up to $40 \%$. Household food security of North-Eastern states of India predominantly depends on rice. Since North Eastern India is home to a wide range of ecological conditions for rice growing in terms of slopes, altitudes, agro climatic conditions, soil types, etc; it has led to immense variability among rice cultivars in the region.

Rice is the staple food of about 3 billion people, nearly half the world's population, depends on rice for survival. In many countries, rice accounts for more than $70 \%$ of human caloric intake and main source of protein for poor people in developing countries. It provides $21 \%$ of global human per capita energy and $15 \%$ of per capita protein (Maclean et al., 2002). Calories from rice are particularly important in Asia, especially among the poor, where it accounts for $50-80 \%$ of daily caloric intake. The major part of rice consists of carbohydrate in the form of starch, which is about 72-75 percent of the total grain composition. The protein content of rice is around 7 percent and the protein of rice contains glutelin, which is also known as oryzenin. The nutritive value of rice protein (biological value $=80$ ) is much higher than that of wheat (biological value $=60$ ) and maize (biological value $=50$ ) or other cereals.
Rice pericarp and germ contain most of minerals including about 4 percent phosphorus. Rice can also be used in cereals, snack foods, brewed beverages, flour, oil, syrup and religious ceremonies to name a few other uses.

Rice production in North East India can be further increased by effective hybridization of locally superior cultivars and elite germplasm, followed by selection in the segregating generations for development of improved high yielding lines suitable to specific agro climatic zones and agronomic practices. Development of varieties adapted to acidic soils is also an important requirement as more than $70 \%$ of the soil in the North East is acidic. As acidic soils suffer from problems of phosphorus deficiency and iron toxicity, it is important to select for improved lines that show tolerance to these stresses. Since low land rice is exposed to many diseases like blast, so breeding of disease resistant varieties, which are the most important part of any Integrated Disease Management (IDM) practice, is required for effective control of disease.

The two pillars of efficient and successful breeding programme are the choice of parental lines and precise selection methodology that can effectively identify transgressive segregants which will lead to increased grain yield per plant and per unit area, eventually leading to development of high yielding varieties. One of the major criteria of parent hybridization programme is the divergence between them with respect to agro-physiological trait.

Researchers have studied complex cause and effect system to determine traits that influence the final grain yield and other important traits during plant ontogeny (Maman et al., 2004, Mohammadi et al., 2003 and Samonte et al., 1998). Yield of paddy is a complex 
quantitative character controlled by many genes interacting with the environment and is the product of many factors called yield components. Selection of parents based on yield alone is often misleading. Hence, the knowledge about relationship between yield and its contributing characters is needed to form an efficient selection strategy for the plant breeders to evolve an economic variety. Grain quality is an economically important trait in rice, and any information about the genetic mechanisms governing grain quality traits will be useful for the rice breeders.

The presence of genetic variability for morphological and yield related traits is of utmost importance for identification and development of desirable genotypes as improvement in any trait is depends on the amount of genetic variability present in the experimental material of that trait. Besides genetic variability, heritability and genetic advance are useful parameters on which selection efficiency depends upon. Heritability is an index of transmissibility of the characters from the parents to offspring and has a predictive role in plant breeding programme. However estimates of heritability alone fail to indicate the response to selection. Therefore estimates of genetic advance along with heritability estimates takes into account for genetic improvement of the selected genotypes over the parental populations for various traits. Thus, the genetic advance has an advantage over heritability and helps breeders in various selection programmes. The genetic advance for the studied traits is dependent on the extent of heritability, genetic variability and selection intensity.

Relatively high heritability and genetic advance values for the traits under study favour the possibility of selection of desirable genotypes. The present investigation was, therefore, undertaken to estimate of genetic variation, heritability and genetic advance in advanced breeding line of low land rice and to identify best genotypes for cultivation under College of post graduate studies, Umiam, Meghalaya in the Kharif season of 2017.

\section{Materials and Methods}

The experiment was carried out at the experimental Farm of College of post graduate studies, CAU (Imphal), Umiam, Meghalaya. The experimental area occupied was uniform in respect of topography and fertility. The climate in Barapani is warm and temperate. In winter, there is much less rainfall in Barapani than in summer. The average annual temperature in Barapani is $20.0{ }^{\circ} \mathrm{C}$. Precipitation here averages 4169 $\mathrm{mm}$. July is the warmest month of the year. The temperature in July averages $23.9{ }^{\circ} \mathrm{C}$. January has the lowest average temperature of the year. It is $13.5^{\circ} \mathrm{C}$. The genotypes included in the study are 22 advanced breeding lines $\left(\mathrm{F}_{7}\right)$ of rice (Oryza sativa) selected based on their yield performance from the previous season. These lines were planted in randomized complete block design with three replications. A detail of genotype are given in Table 1

Experiment consisted of 22 advanced breeding lines and 2 checks lines which were grown in randomized complete block design with three replications. Twenty nine day old seedlings were transplanted in the experimental site with spacing of $20 \mathrm{~cm}$ between plant to plant and $20 \mathrm{~cm}$ between the rows keeping single seedling per hill. Gap filling was done within a week in order to maintain uniform plant population. The standard agronomic practices were adopted for normal crop growth. Observations were recorded as per the DUS guidelines provided by IIRR (Indian Rice Research Institute) Hyderabad. Observations were recorded on the basis of middle five random competitive plants selected from each line in every 
replication for the evaluation of yield and yield contributing traits. Mean of main, average and smallest panicle from each of the five randomly selected plants were used to record the observations of panicle traits. Observations on all the morphological characters were recorded on the net plot basis viz., Basal Leaf sheath color, Leaf Auricle, Leaf Ligule, Ligule shape, Leaf collar, Flag Leaf: Attitude of blade, Leaf sheath anthocyanin colouration, Leaf blade: anthocyanin, Panicle secondary branch, Leaf senescence, Spikelet: color of tip of lemma, Panicle: exsertion, Panicle: awns, Lemma:anthocyanin colouration of area below apex and Observations on all the Quantitative characters were Days to 50 per cent flowering, Plant height $(\mathrm{cm})$, Tillers per plant, Panicle per plant, Panicle length $(\mathrm{cm})$, Leaf length $(\mathrm{cm})$, Leaf width $(\mathrm{cm})$, Leaf area index, Canopy temperature $\left({ }^{0} \mathrm{c}\right)$, Biological yield per plant (g), Spikelets per plant, Number of grains per plant, Spikelet fertility (\%), Harvest Index (\%), 1000- grain weight, Grains yield per plant. Data were compiled by taking mean value over randomly selected plants from all the replications and subjected to the statistical analysis for randomized block design as per Panse and Sukhatme 1984. Genetic parameters such as genotypic (GCV) and phenotypic (PCV) coefficients of variation, heritability and genetic advance were computed as per Burton and De Vane, 1953 and Johnson et al., (1955).

\section{Results and Discussion}

\section{Analysis of variance}

Analysis of variance indicated the existence of significant differences among the genotypes for most of the characters viz., days to $50 \%$ flowering, plant height, Tillers per plant, Panicle per plant, Plant length, Leaf length, Leaf area index, Canopy temperature, Biological yield, Spikelet per plant, No. Of grains per plant, Spikelet fertility, 1000 grain weight and grain yield per plant studied except Leaf width, and Harvest index. The results of analysis of variance are presented in Table 2.

Analysis of variance indicated that mean sum of squares due to genotypes were significant for all the quality traits. This indicates the presence of considerable variability among the breeding lines. Number of grains per plant, spikelet per plant, yield per hectare, leaf area index, days to $50 \%$ flowering, biological yield and plant height he showed maximum variation among breeding lines whereas qualitative traits showed relatively less variation. Padmaja et al., (2008), Khan et al., (2012) and Sahidullah et al., (2009) have also reported highly significant differences for all the characters except flag leaf width and 1000 seed weight among the genotypes. In a similar study, Laxuman et al., (2010) have reported that estimates of genotypic and phenotypic coefficients of variation were high for all the characters except days to fifty per cent flowering and panicle length.

\section{Mean, Genetic variability, heritability}

The genotypic coefficient of variability (GCV) and phenotypic coefficient of variability (PCV), heritability were estimated on the basis of data recorded on twenty four breeding lines including two standard checks. The results obtained for various morphological traits are furnished in Table 3 and mean performance of rice genotypes for various quantitative characters in Table 4.

The characters studied in the present investigation exhibited low, moderate and high PCV and GCV values. Among the metric characters, number of grains per plant recorded highest PCV (28.04) followed by spikelet per plant (24.23) and the lowest PCV (7.16) was recorded for plant height. 
Highest GCV values were recorded for the number of grain per panicle (19.48) followed by spikelet per plant (16.38) whereas lowest GCV value (3.75) was recorded for plant height.

Heritability is classified as low (below 0.30), medium (0.30-0.60) and high (above 0.61). Three characters studied in the present investigation expressed high heritability estimates ranging from 0.62 to 0.99 . Among the metric characters, highest heritability was obtained for leaf length (0.88), followed by leaf width (0.79) and days to $50 \%$ flowering (0.66). Number pf panicles per plant, canopy temperature, number of spikelets per plant and grains per plant showed medium heritability estimates.

A high coefficient of variability indicates that there is a scope of selection and improvement of these traits. High PCV and GCV values were recorded for number of grain per panicle and spikelet per plant which suggests the possibility of improving this trait through selection. The low magnitude of difference between phenotypic and genotypic coefficients of variations were recorded for characters such as days to $50 \%$ flowering, leaf length and leaf width indicating limited influence of environment in the expression of this trait. Thus, selection based on phenotypic expression of the trait would be effective for genetic improvement.

High heritability in broad sense values indicate that the traits under study are less influenced by environment in their expression. Therefore, the quantitative traits are highly heritable. However, highest heritability was recorded for leaf length and leaf width. Moderate heritability estimates were observed for number of panicles per plant, spikelets per panicle, grains per panicle, and spikelet fertility. Unlike our study, high heritability for grain yield plant-1 has been reported by Reddy and De (1996), Reddy et al., (1997), Ashvani et al., (1997), Murthy et al., (1999), Tripathi et al., (1999), Durai et al., (2001), Mishra and Verma (2002), Elayaraja et al., (2004), Hasib et al., (2004), Madhavilatha et al., (2005), Panwar (2005), Girish et al., (2006), Muthuswamy and Ananda Kumar (2006), Narinder (2006), Kole et al., (2008) and Selvaraj et al., (2011). Under low input acidic soil conditions, this was not found to be the case in our study.

Table.1 List of advanced breeding lines and checks used in the study

\begin{tabular}{|l|l|l|}
\hline \multicolumn{2}{|l|}{ Advanced breeding lines } & \multirow{2}{*}{ Checks } \\
\hline CAUS101 & CAUS112 & \\
\hline CAUS102 & CAUS113 & \\
\hline CAUS103 & CAUS114 & \\
\hline CAUS104 & CAUS115 & \multirow{2}{*}{ CAU R1 } \\
\hline CAUS105 & CAUS116 & \multirow{2}{*}{ Shasharang } \\
\hline CAUS106 & CAUS117 & \\
\hline CAUS107 & CAUS118 & \\
\hline CAUS108 & CAUS119 & \\
\hline CAUS109 & CAUS120 & \\
\hline CAUS110 & CAUS121 & \\
\hline CAUS111 & CAUS122 & \\
\hline
\end{tabular}


Table.2 ANOVA for important morphological characters and yield in different rice genotypes

\begin{tabular}{|c|c|c|c|c|c|c|c|c|}
\hline \multirow[t]{2}{*}{ Sl.no. } & \multirow[t]{2}{*}{ Traits } & \multicolumn{2}{|c|}{ MEAN Square } & \multirow[b]{2}{*}{$\begin{array}{c}\text { Total SS } \\
(\mathbf{d f}=71)\end{array}$} & \multirow[b]{2}{*}{ mean value } & \multicolumn{2}{|c|}{ Range } & \multirow[b]{2}{*}{$\mathbf{C V}$} \\
\hline & & Genotype $(\mathrm{df}=23)$ & $\begin{array}{c}\text { Error } \\
(d f=46)\end{array}$ & & & Maximum & Minimum & \\
\hline 1 & DTF & $199.15^{* *}$ & 29.40 & 94.90 & 125.56 & 140.00 & 102.00 & 4.31 \\
\hline 2 & PH & $71.94 * *$ & 33.68 & 50.02 & 95.13 & 105.27 & 85.40 & 6.12 \\
\hline 3 & TPP & $3.16^{* *}$ & 1.52 & 2.01 & 11.43 & 14.00 & 9.40 & 10.87 \\
\hline 4 & PPP & $6.51 * *$ & 2.00 & 3.43 & 9.65 & 12.93 & 6.53 & 14.70 \\
\hline 5 & PL & $6.20 * *$ & 2.96 & 4.06 & 24.77 & 27.67 & 22.67 & 6.97 \\
\hline 6 & $\mathbf{L L}$ & $48.04 * *$ & 2.12 & 17.18 & 34.51 & 41.87 & 28.73 & 4.23 \\
\hline 7 & $\mathbf{L W}$ & 0.15 & 0.01 & 0.07 & 1.80 & 2.27 & 1.53 & 6.09 \\
\hline 8 & LAI & $21350.95 * *$ & 10333.65 & 14177.65 & 537.51 & 742.96 & 380.59 & 18.79 \\
\hline 9 & CT & $4.22 * *$ & 1.34 & 2.36 & 19.86 & 23.64 & 18.19 & 5.80 \\
\hline 10 & BY & $125.86^{* *}$ & 61.82 & 84.42 & 51.33 & 64.53 & 38.60 & 15.24 \\
\hline 11 & SPP & $138257.54 * *$ & 39227.57 & 77044.17 & 1109.02 & 1585.93 & 757.26 & 18.03 \\
\hline 12 & NGPP & $115378.78 * *$ & 30394.70 & 62793.84 & 864.22 & 1250.13 & 510.47 & 20.62 \\
\hline 13 & SF & $92.87 * *$ & 23.39 & 45.33 & 77.92 & 86.09 & 64.12 & 6.21 \\
\hline 14 & HI & 0.06 & 0.02 & 0.04 & 0.71 & 0.93 & 0.41 & 21.94 \\
\hline 15 & $1000 \mathrm{GW}$ & $25.22 * *$ & 11.40 & 16.48 & 24.96 & 32.44 & 19.21 & 13.42 \\
\hline 16 & GYPP & $38.13 * *$ & 17.87 & 29.81 & 21.20 & 29.02 & 15.69 & 20.17 \\
\hline 17 & YPH & $1165583.46^{* *}$ & 409627.08 & 671908.30 & 3699.23 & 4936.00 & 2341.84 & 17.20 \\
\hline \multicolumn{9}{|c|}{$\begin{array}{l}\text { DTF=Days to } 50 \% \text { flowering; PH=Plant height }(\mathrm{cm}) ; \text { TPP=Tillers per plant; PPP=Panicle per plant; PL=Panicle length }(\mathrm{cm}) ; \\
\text { LL=Leaf length }(\mathrm{cm}) ; \mathrm{LW}=\text { Leaf width }(\mathrm{cm}) ; \mathrm{LAI}=\text { Leaf area index; CT=Canopy temperature }\left({ }^{0} \mathrm{c}\right) ; \mathrm{BY}=\mathrm{Biological} \text { yield }(\mathrm{g}) ; \\
\text { SPP=Spikelet per plant; NGPP=No. Of grains per plant; SF=Spikelet fertility }(\%) ; \mathrm{HI}=\text { Harvest index; } 1000 \mathrm{GW}=1000 \text { grain weight } \\
\text { (g); GYPP=grain yield per plant }(\mathrm{g}) ; \mathrm{YPH}=\text { Yield per hectare }(\mathrm{kg}) \text {. } \\
\text { *significant at } 5 \% \text { level of significance, * *significant at } 1 \% \text { level of significance }\end{array}$} \\
\hline
\end{tabular}


Table.3 Components of Variance

\begin{tabular}{|c|c|c|c|c|c|c|c|c|c|c|}
\hline sl.no & character & Vp & Vg & $\begin{array}{c}\text { PCV } \\
(\%)\end{array}$ & $\begin{array}{c}\text { GCV } \\
(\%)\end{array}$ & $\begin{array}{c}\text { ECV } \\
(\%)\end{array}$ & $\mathbf{h}^{2}$ & GA & GG (\%) & $\begin{array}{c}\text { GG/Y } \\
(\%)\end{array}$ \\
\hline 1 & DTF & 85.99 & 56.58 & 7.39 & 5.99 & 4.32 & 0.66 & 15.52 & 13.08 & 1.87 \\
\hline 2 & PH & 46.43 & 12.75 & 7.16 & 3.75 & 6.10 & 0.27 & 7.37 & 7.78 & 1.11 \\
\hline 3 & TPP & 2.07 & 0.55 & 12.59 & 6.46 & 10.80 & 0.26 & 1.52 & 13.25 & 1.89 \\
\hline 4 & PPP & 3.50 & 1.50 & 19.38 & 12.70 & 14.64 & 0.43 & 2.53 & 25.06 & 3.58 \\
\hline 5 & PL & 4.04 & 1.08 & 8.12 & 4.19 & 6.95 & 0.27 & 2.14 & 8.46 & 1.21 \\
\hline 6 & $\mathbf{L L}$ & 17.43 & 15.31 & 12.10 & 11.34 & 4.22 & 0.88 & 8.07 & 23.81 & 3.40 \\
\hline 7 & $\mathbf{L W}$ & 0.06 & 0.04 & 13.19 & 11.70 & 6.09 & 0.79 & 0.44 & 24.70 & 3.53 \\
\hline 8 & LAI & 14006.09 & 3672.43 & 22.02 & 11.27 & 18.91 & 0.26 & 125.02 & 25.03 & 3.58 \\
\hline 9 & CT & 2.30 & 0.96 & 7.63 & 4.93 & 5.83 & 0.42 & 2.02 & 9.41 & 1.34 \\
\hline 10 & BY & 83.16 & 21.35 & 17.77 & 9.00 & 15.32 & 0.26 & 9.53 & 17.57 & 2.51 \\
\hline 11 & SPP & 72237.56 & 33009.99 & 24.23 & 16.38 & 17.86 & 0.46 & 374.82 & 29.53 & 4.22 \\
\hline 12 & NGPP & 58722.73 & 28328.02 & 28.04 & 19.48 & 20.17 & 0.48 & 347.22 & 35.08 & 5.01 \\
\hline 13 & SF & 46.55 & 23.16 & 8.76 & 6.18 & 6.21 & 0.50 & 9.93 & 12.65 & 1.81 \\
\hline 14 & HI & 0.01 & 0.00 & 19.01 & 9.57 & 16.43 & 0.25 & 0.08 & 12.52 & 1.79 \\
\hline 15 & $1000 \mathrm{GW}$ & 16.01 & 4.61 & 16.04 & 8.61 & 13.54 & 0.29 & 4.43 & 17.41 & 2.49 \\
\hline 16 & GYPP & 24.62 & 6.75 & 23.41 & 12.26 & 19.95 & 0.27 & 5.36 & 21.81 & 3.12 \\
\hline 17 & YPH & 661612.54 & 251985.46 & 21.99 & 13.57 & 17.30 & 0.38 & 1035.59 & 29.92 & 4.27 \\
\hline
\end{tabular}


Table.4 Mean performance of rice genotypes for various quantitative characters

\begin{tabular}{|c|c|c|c|c|c|c|c|c|c|c|c|c|c|c|c|c|c|}
\hline Genotype & DTF & PH & TPP & PPP & PL & $\mathbf{L L}$ & LW & LAI & CT & BY & SPP & NGPP & SF & HI & 1000-GW & GYPP & YPH \\
\hline CAUS101 & 128.00 & 89.40 & 14.00 & 12.93 & 24.13 & 41.87 & 1.58 & 454.85 & 18.70 & 52.53 & 1414.00 & 1205.80 & 84.54 & 0.91 & 20.90 & 25.08 & 3826.20 \\
\hline CAUS102 & 17.67 & 105.27 & 9.40 & 6.53 & 27.67 & 34.30 & 2.14 & 505.60 & 19.75 & 46.40 & 918.80 & 775.27 & 84.43 & 0.69 & 26.19 & 20.28 & 2865.28 \\
\hline CAUS103 & 126.67 & 98.53 & 12.33 & 10.40 & 24.00 & 29.47 & 1.66 & 581.43 & 18.87 & 49.93 & 1213.00 & 1002.60 & 79.36 & 0.82 & 23.03 & 23.08 & 3943.82 \\
\hline CAUS104 & 125.67 & 94.67 & 12.53 & 8.07 & 26.07 & 33.00 & 2.09 & 566.17 & 19.60 & 49.53 & 1050.67 & 626.00 & 84.35 & 0.66 & 30.77 & 19.27 & 4520.01 \\
\hline CAUS105 & 130.67 & 94.40 & 10.73 & 8.96 & 25.07 & 36.47 & 1.93 & 455.72 & 20.51 & 54.13 & 1258.27 & 1035.27 & 79.04 & 0.86 & 25.72 & 26.49 & 4710.40 \\
\hline CAUS106 & 127.67 & 93.40 & 10.60 & 8.33 & 24.80 & 35.87 & 1.85 & 626.21 & 18.19 & 48.40 & 1097.73 & 847.40 & 77.31 & 0.82 & 26.33 & 22.17 & 3827.63 \\
\hline CAUS107 & 118.33 & 97.33 & 11.47 & 9.53 & 23.93 & 28.73 & 1.82 & 573.86 & 19.61 & 47.27 & 940.80 & 728.47 & 71.05 & 0.66 & 25.66 & 19.23 & 4170.24 \\
\hline CAUS108 & 116.00 & 95.07 & 11.07 & 10.73 & 22.53 & 39.07 & 1.45 & 742.96 & 21.83 & 51.80 & 1118.13 & 873.73 & 75.83 & 0.69 & 25.21 & 22.05 & 3044.86 \\
\hline CAUS109 & 128.67 & 93.80 & 12.00 & 10.43 & 25.47 & 32.20 & 1.92 & 607.28 & 19.71 & 46.20 & 1094.08 & 824.18 & 75.25 & 0.61 & 21.85 & 18.09 & 3721.37 \\
\hline CAUS110 & 135.33 & 99.93 & 13.13 & 12.13 & 23.73 & 36.67 & 1.67 & 465.78 & 19.09 & 64.53 & 1247.80 & 956.33 & 76.55 & 0.93 & 27.54 & 26.33 & 4936.19 \\
\hline CAUS111 & 140.00 & 95.47 & 12.13 & 11.80 & 23.40 & 37.73 & 1.55 & 506.07 & 18.75 & 50.47 & 899.40 & 687.67 & 76.32 & 0.58 & 23.36 & 16.08 & 3816.80 \\
\hline CAUS112 & 123.00 & 98.67 & 12.60 & 10.73 & 23.67 & 31.00 & 1.85 & 439.48 & 20.51 & 60.40 & 898.80 & 690.00 & 76.76 & 0.60 & 26.66 & 18.39 & 3699.27 \\
\hline CAUS113 & 128.00 & 94.20 & 11.53 & 9.80 & 25.87 & 33.80 & 1.82 & 533.27 & 19.37 & 58.67 & 1382.87 & 1099.27 & 78.12 & 0.83 & 22.11 & 24.29 & 2714.28 \\
\hline CAUS114 & 123.00 & 90.27 & 11.00 & 7.67 & 24.60 & 32.13 & 1.78 & 519.06 & 20.41 & 41.20 & 883.13 & 652.53 & 73.87 & 0.52 & 24.02 & 15.69 & 3282.04 \\
\hline CAUS115 & 133.67 & 98.33 & 11.20 & 9.07 & 27.19 & 30.40 & 1.91 & 629.01 & 19.05 & 52.67 & 1058.33 & 659.07 & 64.12 & 0.57 & 24.28 & 15.99 & 3544.02 \\
\hline CAUS116 & 125.33 & 91.80 & 10.27 & 9.13 & 24.09 & 34.27 & 1.90 & 555.41 & 19.59 & 54.73 & 1490.27 & 1192.33 & 79.86 & 0.79 & 19.20 & 22.87 & 3510.89 \\
\hline CAUS117 & 121.67 & 101.80 & 11.13 & 9.27 & 26.13 & 35.13 & 1.91 & 473.19 & 19.67 & 49.13 & 1026.73 & 868.53 & 84.03 & 0.73 & 24.29 & 21.14 & 3692.93 \\
\hline CAUS118 & 131.33 & 88.80 & 10.67 & 8.73 & 22.67 & 29.07 & 1.39 & 541.82 & 20.83 & 38.60 & 887.33 & 765.20 & 86.09 & 0.61 & 24.66 & 18.85 & 3621.39 \\
\hline CAUS119 & 129.33 & 93.47 & 11.13 & 9.47 & 23.07 & 43.73 & 2.27 & 666.85 & 20.13 & 57.80 & 1318.77 & 964.73 & 73.48 & 0.75 & 23.22 & 22.38 & 3538.31 \\
\hline CAUS120 & 132.00 & 100.07 & 10.00 & 8.33 & 25.73 & 36.50 & 2.08 & 531.23 & 18.57 & 46.27 & 1105.87 & 892.27 & 80.83 & 0.81 & 24.73 & 22.05 & 3815.93 \\
\hline CAUS121 & 132.00 & 93.73 & 11.07 & 9.07 & 25.27 & 38.33 & 1.68 & 430.50 & 19.71 & 50.00 & 1016.07 & 904.67 & 84.58 & 0.79 & 25.52 & 23.19 & 4715.05 \\
\hline CAUS122 & 102.00 & 85.40 & 11.27 & 10.38 & 24.67 & 30.80 & 1.53 & 495.31 & 21.23 & 40.63 & 757.26 & 510.47 & 67.23 & 0.41 & 32.43 & 16.54 & 2341.84 \\
\hline CAU R1 & 112.00 & 87.47 & 11.00 & 9.16 & 23.40 & 30.53 & 1.82 & 380.59 & 23.64 & 45.83 & 952.53 & 729.36 & 75.86 & 0.45 & 27.69 & 20.15 & 3563.17 \\
\hline Shasharang & 125.33 & 101.87 & 12.00 & 11.03 & 27.27 & 37.27 & 1.71 & 618.50 & 19.29 & 62.67 & 1585.93 & 1250.13 & 81.15 & 0.86 & 23.16 & 29.02 & 3359.51 \\
\hline SEM & 3.36 & 3.37 & 0.61 & 0.70 & 0.85 & 0.76 & 0.10 & 26.75 & 0.53 & 4.02 & 102.50 & 93.34 & 2.00 & 0.14 & 1.04 & 2.55 & 376.85 \\
\hline
\end{tabular}


Table.5 Characterization of rice genotypes with respect to discreet characters

\begin{tabular}{|c|c|c|c|c|c|c|c|c|c|c|c|c|c|c|}
\hline & BLSC & LA & $\mathbf{L L}$ & $\mathbf{L S}$ & LC & FLAB & LSAC & LBA & PSB & $\mathbf{L S}$ & SCTL & PE & PA & LABA \\
\hline CAUS101 & Green & Present & Present & Cleft type & Absent & Semi-erect & Green & Absent & Present & Late & White & Well exserted & Absent & Absent \\
\hline CAUS102 & Green & Present & Present & Cleft type & Absent & Erect & Green & Absent & Present & Early & Purple & Partly & Absent & Present \\
\hline CAUS103 & Green & Present & Present & Cleft type & Absent & Erect & Green & Absent & Present & Medium & Brown & Partily & Absent & Absent \\
\hline CAUS104 & Green & Present & Present & Cleft type & Present & Erect & Green & Absent & Present & Medium & Purple & Well exserted & Absent & Present \\
\hline CAUS105 & Green & Present & Present & Cleft type & Absent & Erect & Green & Absent & Present & Late & Yellow & Partily & Absent & Absent \\
\hline CAUS106 & Green & Present & Present & Cleft type & Absent & Erect & Green & Absent & Present & Late & Brown & Well exserted & Absent & Absent \\
\hline CAUS107 & Green & Present & Present & Cleft type & Absent & Erect & Green & Absent & Present & Early & Yellow & Partily & Absent & Absent \\
\hline CAUS108 & Green & Present & Present & Cleft type & Absent & Erect & Green & Absent & Present & Early & White & Partily & Absent & Absent \\
\hline CAUS109 & Green & Present & Present & Cleft type & Absent & Erect & Green & Absent & Present & Medium & Yellow & Well exserted & Absent & Absent \\
\hline CAUS110 & Green & Present & Present & Cleft type & Absent & Erect & Green & Absent & Present & Late & Brown & Partily & Absent & Absent \\
\hline CAUS111 & Green & Present & Present & Cleft type & Absent & Horizontal & Green & Absent & Present & Late & Brown & Partily & Absent & Absent \\
\hline CAUS112 & purple & Present & Present & Cleft type & Absent & Erect & purple & Absent & Present & Early & Purple & Partily & Absent & Present \\
\hline CAUS113 & Green & Present & Present & Cleft type & Absent & Erect & Green & Absent & Present & Medium & White & Well exserted & Absent & Absent \\
\hline CAUS114 & Green & Present & Present & Cleft type & Absent & Erect & Green & Absent & Present & Medium & Purple & Partily & Absent & Present \\
\hline CAUS115 & purple & Present & Present & Cleft type & Present & Semi-erect & purple & Absent & Present & Late & & & Absent & Present \\
\hline CAUS116 & Green & Present & Present & Cleft type & Absent & Semi-erect & Green & Absent & Present & Medium & White & Partily & Absent & Absent \\
\hline CAUS117 & Green & Present & Present & Cleft type & Absent & Erect & Green & Absent & Present & Medium & White & Partily & Absent & Absent \\
\hline CAUS118 & purple & Present & Present & Cleft type & Present & Erect & purple & Absent & Present & Medium & Purple & Partily & Absent & Present \\
\hline CAUS119 & purple & Present & Present & Cleft type & Present & Erect & purple & Absent & Present & Early & White & Partily & Absent & Absent \\
\hline CAUS120 & purple & Present & Present & Cleft type & Present & Semi-erect & purple & Present & Present & Medium & Purple & Partily & Absent & Present \\
\hline CAUS121 & Green & Present & Present & Cleft type & Absent & Erect & Green & Absent & Present & Medium & Yellow & Well exsertion & Absent & Absent \\
\hline CAUS122 & Green & Present & Present & Cleft type & Absent & Erect & Green & Absent & Present & Early & White & Partily & Absent & Absent \\
\hline CAU R1 & Green & Present & Present & Cleft type & Absent & Erect & Green & Absent & Present & Early & White & Partily & Absent & Absent \\
\hline Shasharang & Green & Present & Present & Cleft type & Absent & Erect & Green & Absent & Present & Early & White & Partily & Absent & Absent \\
\hline
\end{tabular}

BLSC=Basal Leaf sheath colour ; LA=Leaf Auricle; LL=Leaf Ligule ; LS=Ligule shape; LC=Leaf collar ; FLAB=Flag Leaf: Attitude of blade ; LSAC=Leaf sheath anthocyanin colouration ;LBA=Leaf blade: anthocyanin ; PSB=Panicle secondary branch; LS= Leaf senescence ;SCTL Spikelet: color of tip of lemma ; PE=Panicle: exserted ; PA=Panicle: awns ; LABA=Lemma:anthocyanin colouration of area below apex 
Fig.(A) Blast Disease Scoring; (B) Bronzing Scoring

(A)

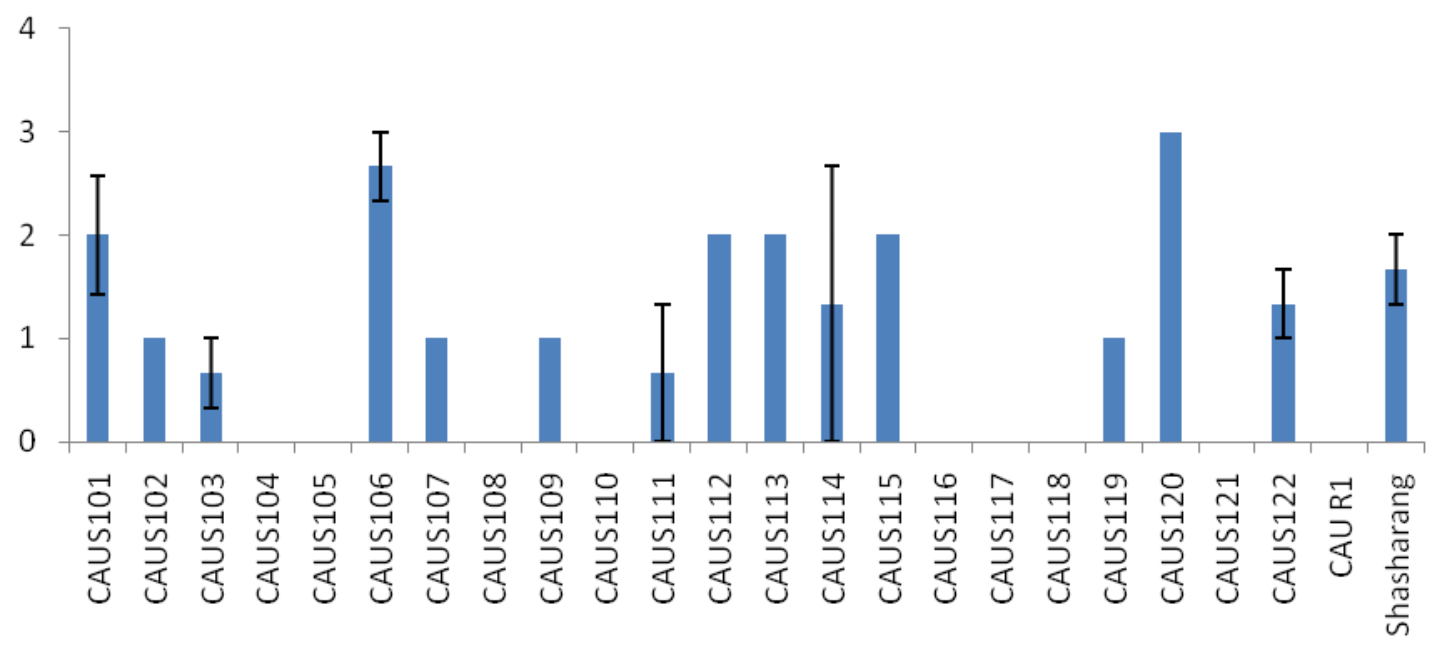

(B)

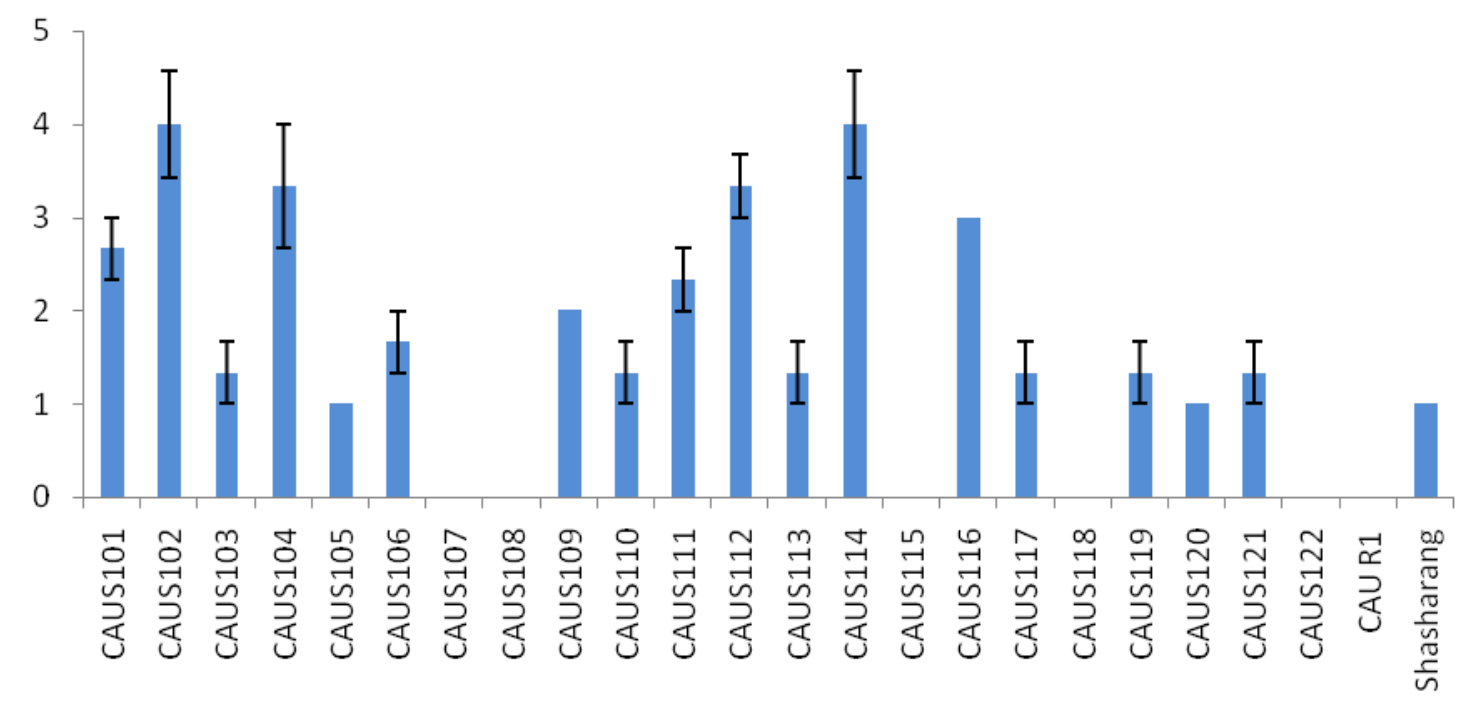

\section{DUS characterization}

Twenty four genotypes were characterized using seventeen morphological characters as per standard evaluation system (IRRI, 1996) (Table 5). These descriptors are highly heritable, unambiguous and easily identifiable. The study of morphological 
traits was carried to describe each genotype and establish their diagnostic characteristics. The experimental material showed great variability for the sixteen morphological traits (Table 5). All the traits viz. basal leaf sheath colour, Leaf Auricle, Leaf Ligule, Ligule shape, Leaf collar, Flag Leaf: Attitude of blade, Leaf sheath anthocyanin colouration, leaf blade: anthocyanin, Panicle secondary branch, Stem thickness, Leaf senescence, Spikelet: color of tip of lemma, Panicle: exsertion, Panicle: awns, Lemma: anthocyanin colouration of area below apex, Resistance to blast Disease Scoring 0 to 5 . (Fig.A), Bronzing Scoring 0 to 5. (Fig.B) exhibited wide variation among the genotypes under study.

In conclusion, analysis of variance indicated that mean sum of squares due to genotypes were significant for all the quality traits. This indicates the presence of considerable variability among the breeding lines. Number of grains per plant, spikelet per plant, yield per hectare, leaf area index, days to $50 \%$ flowering, biological yield and plant height showed maximum variation among breeding lines whereas qualitative traits showed relatively less variation. A high coefficient of variability indicates that there is a scope of selection and improvement of these traits. High PCV and GCV values were recorded for number of grain per panicle and spikelet per plant which suggests the possibility of improving this trait through selection. The low magnitude of difference between phenotypic and genotypic coefficients of variations were recorded for characters such as days to $50 \%$ flowering, leaf length and leaf width indicating limited influence of environment in the expression of this trait. Thus, selection based on phenotypic expression of the trait would be effective for genetic improvement. High heritability in broad sense values indicate that the traits under study are less influenced by environment in their expression. Therefore, the quantitative traits are highly heritable. However, highest heritability was recorded for leaf length and leaf width.

\section{References}

Ashvani, P., Dhaka R.P.S., Sharma, R.K. and Arya, K.P.S. 1997. Genetic variability and inter-relationship in rice (Oryza sativa L.). Adv. Plant Sci., 10(1): 29-32.

Durai, A.A., Ngachan, S.V., Pattanayak, A., and Sarma, B.K. 2001. Comparative study of heritability, genetic advance and association of characters in conventionally breed and doubled haploid lines of rice (Oryza sativa L.). Indian J. Hill Farming, 14(2): 71-75.

Elayaraja, K., Prakash, M., Saravanan, K., Kumar, B.S., and Ganesan, J. 2004. Studies on variability and heritability in M2 generation of rice (Oryza sativa L.). Res. Crops, 5(2/3): 240-242.

Girish, T.N., Gireesha, T.M., Vaishali, M.G., Hanamareddy, B.G., and Hittalmani, S. 2006. Response of a new IR50/Moroberekan recombinant inbred population of rice (Oryza sativa L.) from an Indica $\mathrm{x}$ Japonica cross for growth and yield traits under aerobic conditions. Euphytica, 152 (2): 149-161.

Hasib, K.M., Ganguli, P.K., and Kole, P.C. 2004. Evaluation of the performance of advanced generation lines of mutant $\mathrm{x}$ Basmati crosses of scented rice. $J$. Interacademicia, 8(1): 7-10.

IRRI 1996. Standard evaluation system for rice. Int. Rice Res. Institute, Manila, Philippines.

Khan, M.A., Khan, A.S., Khan, U., Habib, S., and Ahmad, R. 2012. Association of various morphological traits with yield and genetic divergence in rice (Oryza sativa L.). Int. J. Agric. Biol., 14(1): 55-62.

Kole, P.C., Chakraborty, N.R., and Bhat, J.S. 2008. Analysis of variability, correlation and path coefficients in induced mutants of aromatic non-basmati rice. Tropical Agric. Res. and Ext., 11: 60-64.

Laxuman, L., Salimath, P., Shashidhar, H., Mohankumar, H.D., Patil, S.S., 
Vamadeviha, H.M., and Janagoudar, B.S. 2010. Analysis of genetics variability in interspecific backcross inbred lines in rice (Oryza sativa L.). Karnataka J. Agric. Sci., 23(4): 563-565.

Maclean, J., Dawe, D., Hardy, B., and Hettel, G.P. 2002. Rice Almanac. Los Baños, Philippines, International Rice Research Institute (IRRI); Bouaké, Côte d'Ivoire, West Africa Rice Development Association; Cali, Colombia. International Center for Tropical Agriculture.

Madhavilatha, L., Sekhar, M.R., Suneetha, Y., and Srinivas, T. 2005. Genetic variability, correlation and path analysis for yield and quality traits in rice (Oryza sativa L.). Res. Crops., 6(3): 527-534.

Maman, N., Mason, S.C., Lyon, D.J. and Dhungana, P. 2004. Yield components of pearl millet and grain sorghum across environments in the Central Great Plains. Crop Sci., 44(6): 2138-2145.

Mishra, L.K. and Verma, R.K. 2002. Genetic variability for quality and yield traits in nonsegregating populations of rice (Oryza sativa L.). Plant Archives, 2 (2): 251-256.

Mohammadi, S.A., Prasanna, B.M., and Singh, N.N. 2003. Sequential path model for determining interrelationships among grain yield and related characters in maize. Crop sci., 43(5): 1690-1697.

Murthy, N., Kulkarni, R.S., and Udaykumar, M. 1999. Genetic variability, heritability and genetic advance for morphological traits in rice. Oryza, 36(2): 159-160.

Muthuswamy, A. and Ananda, K. 2006. Variability studies in drought resistant cultures of rice. Res. Crops, 7(1): 130-132.

Narinder 2006. Phenotyping of Indica-Japonica derived RILs of Rice for Quantitative traits. Ph.D. Thesis, Submitted to JNKVV,

\section{How to cite this article:}

Ashish Rai, Mayank Rai, Bidisha Borpatragohain and Shivendra Kumar. 2020. Assessment of Genetic Variability, Heritability and Genetic Advance for Yield in Advanced Breeding Line (Oryza sativa L.) of Low Land Rice in Meghalaya. Int.J.Curr.Microbiol.App.Sci. 9(03): 706717. doi: https://doi.org/10.20546/ijcmas.2020.903.085
Jabalpur.
Padmaja, D., Radhika, K., Subba Rao, L.V., and Padma, V. 2008. Studies on variability, heritability and genetic advance for quantitative characters in rice (Oryza sativa L.). Indian J. Plant Genet. Res., 21(3): 7184.

Panwar, L.L. 2005. Genetic variability, heritability and genetic advance for panicle characters in transplanted rice. Res. Crops, 6(3): 505-508.

Reddy, J.N. and De, R.N. 1996. Genetic variability in lowland rice. Madras Agric.

Reddy, J.N., Pani, D., and Roy, J.K. 1997. Variability and character association in lowland rice. Indian Agriculturist, 41(3): 159-65.

Sahidulla, S.M., Hanafi, M.M., Ashrafuzzaman, M., Ismail, M.R., and Khair, A. 2009. Genetic diversity in grain quality and nutrition of aromatic Rice. Afr. J. Biot., 8(7): 1238-1246.

Samonte, S.O., Wilson, L.T., and McClung, A.M. 1998. Path analysis of yield and yield related traits of fifteen diverse rice genotypes. Crop Sci., 38: 1130-1136.

Selvaraj, C.I., Pothiraj, N., Thiyagarajan, K., Bharathi, M., and Rabindran, R. 2011. Genetic parameters of variability, correlation and path coefficient studies for grain yield and other yield attributes among rice blast disease resistant genotypes of rice (Oryza sativa L.). African J. Biotech., 10(17): 3322-3334.

Tripathi, A.K., Sinha, S.K., and Bhandarkar, S. 1999. Studies on variability, heritability and genetic advance of semideep water rice. Adv. Plant. Sci., 12 (1): 233-235. J., 83(4): 269-270. 